The short intermolecular contact $(\mathrm{C}-) \mathrm{H}(3) \cdots \mathrm{O}(24)$, 2.47 (5) $\AA\left|\frac{1}{2}+x, \frac{1}{2}-y, z\right|$, with $d=0.23 \AA$ (Taylor \& Kennard, 1982) probably forms a weak hydrogen bond; otherwise intermolecular distances correspond to or are greater than the relevant van der Waals radii.

Thanks are due to Professor S. N. Sawhney of this Department for the crystal sample and to Professor W. H. Watson, Chemistry Department, Texas Christian University, Fort Worth, USA, for his kind help.

\section{References}

Abignente, E., Arena, F., de Caparis, P. \& Parente, L. (1976). Farmaco Ed. Sci. 31, 880-883.

argay, Gy., Kálmán, A., Lazar, D., Ribar,, B. \& Tóth, G. (1977). Acta Cryst. B 33, 99-105.

Cameron, A. F., Cameron, I. R. \& Duncanson, F. D. (1981). J. Chem. Soc. Perkin Trans. 2, pp. 789-791.

Cromer, D. T. \& ManN, J. B. (1968). Acta Cryst. A24, 321-324.

Fibiger, R. F., Banks, A. R., Jones, T., Haltiwanger, R. C. \& W ATt, D. S. (1978). J. Heterocycl. Chem. 15, 307-310.

Foresti, E., Sanseverino, L. R. D. \& Sabatino, P. (1985). Acta Cryst. C41, 240-243.

International Tables for X-ray Crystallography (1974). Vol. IV. Birmingham: Kynoch Press. (Present distributor D. Reidel, Dordrecht.)
JoHNSON, C. K. (1971). ORTEPII. Report OR NL-3794, revised. Oak Ridge National Laboratory, Tennessee, USA.

Kornis, G., Marks, P. J. \& Chidester, C. G. (1980). J. Org. Chem. 45, 4860-4863.

Lambardino, J. G. (1978). Annual Reports in Medicinal Chemistry, Vol. XIII, pp. 167-168. New York: Academic Press.

Main, P., Hull, S. E., Lessinger, L., Germain, G., Declerce, J.-P. \& Woolfson, M. M. (1978). MULTAN78. A System of Computer Programs for the Automatic Solution of Crystal Structures from X-ray Diffraction Data. Univs. of York, England, and Louvain, Belgium.

Mathew, M. \& Palenik, G. J. (1974). J. Chem. Soc. Perkin Trans. 2, pp. 532-536.

Raeymakers, A. H. M., Roevens, L. F. C. \& Janssen, P. A. J. (1967). Tetrahedron Lett. pp. 1467-1470.

Sawhney, S. N., Kodali, D. R., Dhindsa, G. S. \& Singh, S. P. (1982). Ind. J. Chem. 21B, 134-138.

Senda, H., Matsuoka, H. \& Maruha, J. (1986). Acta Cryst. C42, $1087-1089$.

Stewart, J. M., Machin, P. A., Dickinson, C. W., Ammon, H. L., HeCK, H. \& FlaCK, H. (1976). The XRAY76 System. Tech. Rep. TR-446. Computer Science Center, Univ. of Maryland, College Park, Maryland, USA.

Stewart, R. F., Davidson, E. R. \& Simpson, W. T. (1965). J. Chem. Phys. 42, 3175-3187.

Taylor, R. \& Kennard, O. (1982). J. Am. Chem. Soc. 104, 5063-5070.

WILKINS, S. A. JR. \& OlkowsKI, Z. L. (1977). Cancer, 39, 487-489.

Acta Cryst. (1987). C43, 2418-2420

\title{
Structures of Two Isomeric Phenylethanolamine Analogs Containing the Benzobicyclo- [3.2.1]octane Skeleton
}

\author{
By GaRY L. GRUNewALd AND QIzHUANG Ye \\ Department of Medicinal Chemistry, University of Kansas, Lawrence, Kansas 66045, USA \\ and Fusao Takusagawa \\ Department of Chemistry, University of Kansas, Lawrence, Kansas 66045, USA
}

(Received 20 May 1987; accepted 3 September 1987)

\begin{abstract}
Amino-6,7,8,9-tetrahydro-5,8methano-5H-benzocyclohepten-9-ol hydrochloride, $\mathrm{C}_{12} \mathrm{H}_{16} \mathrm{NO}^{+} . \mathrm{Cl}^{-}, M_{r}=225 \cdot 72$, tetragonal, I4 $/ a, a$ $=b=24.641$ (3), $c=7.709$ (1) $\AA, \quad V=4681$ (1) $\AA^{3}$, $Z=16, D_{x}=1.281 \mathrm{Mg} \mathrm{m}^{-3}, \lambda(\mathrm{Mo} K \alpha)=0.71069 \AA$, $\mu=0.3032 \mathrm{~mm}^{-1}, \quad F(000)=1920, \quad T=297 \mathrm{~K} . \quad R=$ 0.0324 for 1543 independent reflections collected. exo-8-Amino-6,7,8,9-tetrahydro-5,8-methano-5 $\mathrm{H}$ -

benzocyclohepten-9-ol, $\quad \mathrm{C}_{12} \mathrm{H}_{15} \mathrm{NO}, \quad M_{r}=189 \cdot 26$, monoclinic, $P 21 / c, a=13.863$ (3), $b=6.910$ (2), $c$ $=13.437$ (3) $\AA, \quad V=1018.2(5) \AA^{3}, \quad Z=4, \quad D_{x}=$ $1.235 \mathrm{Mg} \mathrm{m}^{-3}, \quad \lambda($ Mo $K \alpha)=0.71069 \AA, \quad \mu=$ $0.08456 \mathrm{~mm}^{-1}, F(000)=408, T=297 \mathrm{~K} . R=0.0378$ for 1340 independent reflections collected. The torsion angles $\mathrm{N}-\mathrm{C}(8)-\mathrm{C}(9)-\mathrm{C}(9 \mathrm{a})$ of the two isomers are
\end{abstract}

0108-2701/87/122418-03\$01.50
174.0 (2) and $173.4(1)^{\circ}$, respectively, which are very close to those observed for epinephrine $\left(172^{\circ}\right)$ and norepinephrine $\left(179^{\circ}\right)$ with the side chain in a fully extended conformation. The cyclohexene moiety has a half-chair conformation as found in tetralols.

Introduction. In the continuation of our effort in studying the relationship between conformation and biological activities in phenylethanolamine analogs (Grunewald, Reitz, Hallett, Rutledge, Vollmer, Archuleta \& Ruth, 1980; Rafferty \& Grunewald, 1982; Grunewald, Pleiss \& Rafferty, 1982), we obtained endo- and exo-8-amino-6,7,8,9-tetrahydro-5,8methano-5H-benzocyclohepten-9-ols (1) and (2). They are both conformationally defined analogs of phenyl-

c 1987 International Union of Crystallography 
ethanolamine. In order to assign the stereochemistry of the hydroxyl group in the two isomers unambiguously, we carried out $\mathrm{X}$-ray crystallographic studies on each isomer.

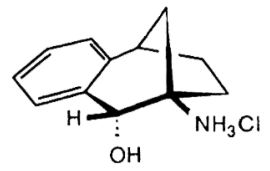

(1)

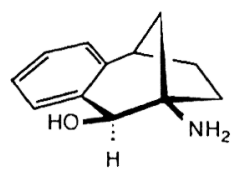

(2)
Experimental. endo-8-Amino-6,7,8,9-tetrahydro-5,8methano- $5 H$-benzocyclohepten-9-ol hydrochloride [compound (1)] was obtained by recrystallization from

Table 1. Summary of data-collection and processing parameters

$\begin{array}{lll} & \text { Compound (1) } & \text { Compound (2) } \\ \text { Crystal size (mm) } & 0.3 \times 0.3 \times 0.5 & 0.3 \times 0.3 \times 0.1 \\ \text { Range of } h k l & -26 \rightarrow 26,0 \rightarrow 26,0-8 & 0 \rightarrow 14,0 \rightarrow 7,-14-\cdots 10 \\ R_{\text {int }} & 0.018 & 0.029 \\ \text { Total reflections } & 3891 & 2857 \\ \text { Independent reflections } & 1543 & 1340 \\ \text { Total variables } & 201 & 188 \\ R & 0.0324 & 0.0378 \\ w R & 0.0314 & 0.0493 \\ S & 1.182 & 0.9770\end{array}$

Table 2. Fractional coordinates with e.s.d.'s in parentheses and equivalent isotropic thermal parameters $\left(\AA^{2}\right)$ for non-H atoms

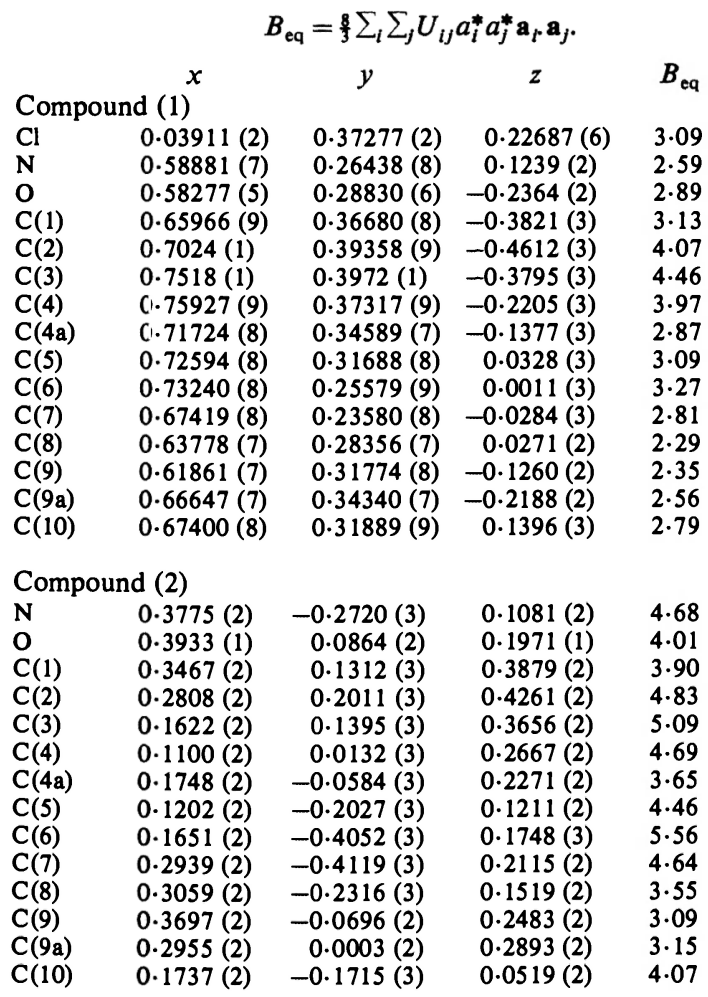

ethanol and ether as colorless tetragonal prisms and exo-8-amino-6,7,8,9-tetrahydro-5,8-methano- $5 \mathrm{H}$ -

benzocyclohepten-9-ol [compound (2)] was recrystallized from methylene chloride as colorless plates. Each crystal was mounted on a glass fiber oriented approximately along [001] for (1) or [100] for (2). Cell constants were determined using 15 centered reflections widely scattered throughout reciprocal space. Full spheres of data out to $2 \theta=45^{\circ}$ were collected using a Syntex $P 2{ }_{1}$ diffractometer (Mo $K \alpha$, graphite monochromator) with $\theta-2 \theta$ scan mode. Corrections were made in intensity of two [for (2)] or three [for (1)] standard reflections monitored every 100 measurements. Independent $F_{o}^{2}$ data were obtained by merging equivalent reflections. The reflections with $F_{o}^{2}<$ $0.2 \sigma\left(F_{o}^{2}\right)$ were reset to $F_{o}^{2}=0.2 \sigma\left(F_{o}^{2}\right)$. All reflections were used in subsequent calculations. The structures were solved by the direct method using MULTAN78 (Main, Hull, Lessinger, Germain, Declercq \& Woolfson, 1978). All H atoms were located from the difference Fourier maps. Full-matrix refinement, anisotropic for non- $\mathrm{H}$ and isotropic for $\mathrm{H}$ atoms. Function minimized $\sum w\left(\left|F_{o}\right|-\left|F_{c}\right|\right)^{2}$ with $w=1 / \sigma^{2}\left(F_{o}\right)$. The final difference Fourier maps were featureless $\left[ \pm 0.17 \mathrm{e} \AA^{-3}\right.$ for (1) and $\pm 0.26 \mathrm{e} \AA^{-3}$ for (2)]. The $\Delta / \sigma$ of the last cycle of least squares was less than 0.13 for (1) and 0.76 for (2). Atomic scattering factors were taken from International Tables for $X$-ray Crystallography

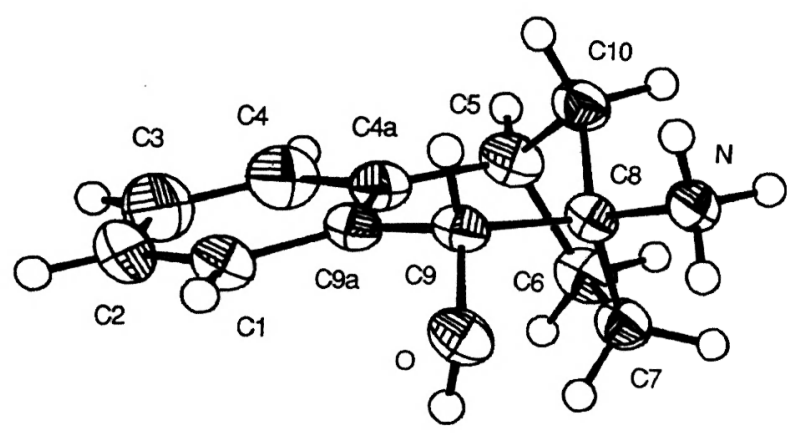

(1)

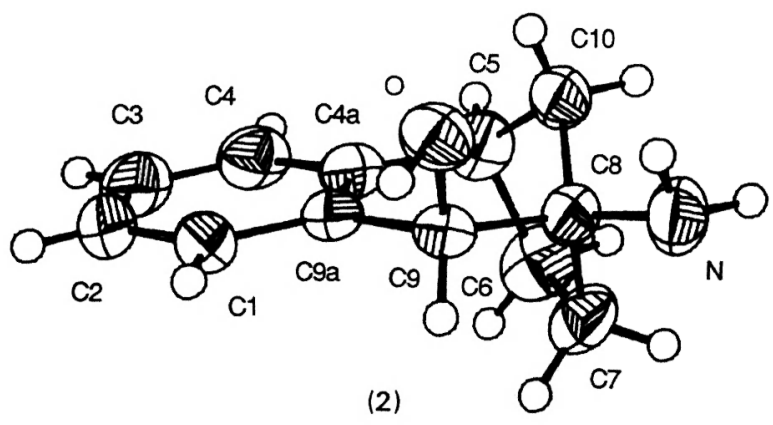

Fig. 1. ORTEPII (Johnson, 1976) drawings of (1) and (2). Non-H atoms are displayed as principal ellipses at the $50 \%$ probability level, $\mathrm{H}$ atoms as small spheres of arbitrary size. 
Table 3. Bond lengths $(\AA)$, bond angles $\left(^{\circ}\right)$ 'and torsion angles $\left(^{\circ}\right)$ involving non- $\mathrm{H}$ atoms, with their e.s.d.'s in parentheses

$\mathrm{N}-\mathrm{C}(8)$

O-C $(9)$

$C(1)-C(2)$
$C(1)-C(9 a)$

$\mathrm{C}(2)-\mathrm{C}(3)$

$\mathrm{C}(3)-\mathrm{C}(4)$

$\mathrm{C}(2)-\mathrm{C}(1)-\mathrm{C}(9 \mathrm{a})$

$C(1)-C(2)-C(3)$

$C(2)-C(3)-C(4)$

C (3)-C(4)-C(4a)

C (4) $-C(4 a)-C(5)$

C (5)-C(4a)-C $(9 a)$

$C(4 a)-C(5)-C(6)$

$C(4 a)-C(5)-C(10)$

$\mathrm{C}(9 \mathrm{a})-\mathrm{C}(1)-\mathrm{C}(2)-\mathrm{C}(3)$

$C(2)-C(1)-C(9 a)-C(4 a)$

$\mathrm{C}(2)-\mathrm{C}(1)-\mathrm{C}(9 \mathrm{a})-\mathrm{C}(9)$

C(1)-C(2)-C(3)-C(4)

$C(2)-C(3)-C(4)-C(4 a)$

$C(3)-C(4)-C(4 a)-C(5)$

$\mathrm{C}(3)-\mathrm{C}(4)-\mathrm{C}(4 \mathrm{a})-\mathrm{C}(9 \mathrm{a})$

C (4)-C(4a)-C(5)-C(6)

$C(4)-C(4 a)-C(5)-C(10)$

$C(9 a)-C(4 a)-C(5)-C(6)$

$C(9 a)-C(4 a)-C(5)-C(10)$

$C(4)-C(4 a)-C(9 a)-C(1)$

\begin{tabular}{rr}
$\begin{array}{c}\text { Com- } \\
\text { pound } \\
(1)\end{array}$ & \multicolumn{1}{c}{$\begin{array}{c}\text { Com- } \\
\text { pound }\end{array}$} \\
$1.495(2)$ & \multicolumn{1}{c}{$1.458(4)$} \\
$1.425(2)$ & $1.421(3)$ \\
$1.384(3)$ & $1.382(5)$ \\
$1.395(3)$ & $1.388(3)$ \\
$1.375(4)$ & $1.382(4)$ \\
$1.373(4)$ & $1.369(3)$ \\
$120.2(2)$ & $120.9(2)$ \\
$120.2(2)$ & $119.6(2)$ \\
$120.0(2)$ & $120.0(3)$ \\
$121.2(2)$ & $121.2(2)$ \\
$121.5(2)$ & $122.0(2)$ \\
$118.9(2)$ & $119.2(2)$ \\
$119.6(2)$ & $118.8(2)$ \\
$109.9(2)$ & $109.7(2)$ \\
$109.6(2)$ & $109.5(2)$ \\
$0.2(3)$ & $0.2(3)$ \\
$-1.8(3)$ & $1.0(3)$ \\
$175.4(2)$ & $178.7(2)$ \\
$1.4(4)$ & $-1.4(3)$ \\
$-1.2(4)$ & $1.4(3)$ \\
$176.7(2)$ & $178.0(2)$ \\
$-0.4(3)$ & $-0.2(3)$ \\
$-100.5(2)$ & $-100.2(2)$ \\
$148.3(2)$ & $148.6(2)$ \\
$76.6(2)$ & $78.0(2)$ \\
$-34.6(2)$ & $-33.3(2)$ \\
$1.9(3)$ & $-1.0(3)$
\end{tabular}

C(4)-C(4a)

$C(4 a)-C(5)$

$C(4 a)-C(9 a)$

$\mathrm{C}(5)-\mathrm{C}(6)$

$\mathrm{C}(5)-\mathrm{C}(10)$

$\mathrm{C}(6)-\mathrm{C}(5)-\mathrm{C}(10)$

$\mathrm{C}(5)-\mathrm{C}(6)-\mathrm{C}(7)$

$\mathrm{C}(6)-\mathrm{C}(7)-\mathrm{C}(8)$

$\mathrm{N}-\mathrm{C}(8)-\mathrm{C}(7)$

$\mathrm{N}-\mathrm{C}(8)-\mathrm{C}(9)$

$\mathrm{N}-\mathrm{C}(8)-\mathrm{C}(10)$

$\mathrm{C}(7)-\mathrm{C}(8)-\mathrm{C}(9)$

$\mathrm{C}(7)-\mathrm{C}(8)-\mathrm{C}(10)$

C(4)-C(4a)-C(9a)-C(9)

$C(5)-C(4 a)-C(9 a)-C(1)$

$C(5)-C(4 a)-C(9 a)-C(9)$
$C(9)-C(5)-C(6)-C(7)$

C (4a)-C(5)-C(6)-C(7)

$C(10)-C(5)-C(6)-C(7)$

$C(4 a)-C(5)-C(10)-C(8)$

$\mathrm{C}(6)-\mathrm{C}(5)-\mathrm{C}(10)-\mathrm{C}(8)$

$\mathrm{C}(5)-\mathrm{C}(6)-\mathrm{C}(7)-\mathrm{C}(8)$

$\mathrm{C}(6)-\mathrm{C}(7)-\mathrm{C}(8)-\mathrm{N}$

$\mathrm{C}(6)-\mathrm{C}(7)-\mathrm{C}(8)-\mathrm{C}(9)$

$C(6)-C(7)-C(8)-C(10)$

$\mathrm{N}-\mathrm{C}(8)-\mathrm{C}(9)-\mathrm{O}$

$\begin{array}{rr}\begin{array}{r}\text { Com } \\ \text { pound } \\ (1)\end{array} & \begin{array}{r}\text { Com } \\ \text { pound } \\ (2)\end{array} \\ 1.390(3) & 1.386(4) \\ 1.512(3) & 1.509(3) \\ 1.400(3) & 1.397(3) \\ 1.533(3) & 1.522(3) \\ 1.522(3) & 1.518(4) \\ & \\ 101.9(2) & 102.1(2) \\ 104.0(2) & 103.8(2) \\ 105.0(2) & 106.4(2) \\ 111.5(2) & 111.7(2) \\ 108.1(1) & 107.5(2) \\ 111.8(2) & 116.4(2) \\ 112.8(2) & 110.1(2) \\ 104.7(2) & 103.0(2) \\ & \\ -175.3(2) & -178.6(2) \\ -175.3(2) & -179.2(2) \\ 7.5(3) & 3.2(3) \\ -77.9(2) & -78.9(2) \\ 38.3(2) & 37.1(2) \\ 66.9(2) & 67.4(2) \\ -49.5(2) & -48.8(2) \\ -11.9(2) & -11.7(2) \\ -139.9(2) & -143.6(2) \\ 98.2(2) & 97.0(2) \\ -18.9(2) & -17.9(2) \\ -57.1(2) & 51.1(2)\end{array}$

$\mathrm{C}(7)-\mathrm{C}(8)$

$\mathrm{C}(8)-\mathrm{C}(9)$

$C(8)-C(10)$

C (9)-C(9a)

Com-

pound

(1)

$1.534(3)$

$1.525(3) \quad 1.521(2)$

$1.519(3) \quad 1.524(2)$

$\begin{array}{ll}1.517(3) & 1.512(4)\end{array}$

C(9)-C(8)-C(10)

$\mathrm{O}-\mathrm{C}(9)-\mathrm{C}(8)$

O-C (9)-C(9a)

C (8)-C(9)-C(9a)

$C(1)-C(9 a)-C(4 a)$

$C(1)-C(9 a)-C(9)$

C $(4 a)-C(9 a)-C(9)$

$\mathrm{C}(5)-\mathrm{C}(10)-\mathrm{C}(8)$

$107.9(2) \quad 108.0(2)$

$111.9(2) \quad 109.3(2)$

$114.4(2) \quad 110.1(2)$

110.7 (1) $\quad 112.0(2)$

$119.5(2) \quad 119.1(2)$

$120.3(2) \quad 119.3(2)$

$120.2(2)$

$120.2(2)$
$99.6(2) \quad 101.3(2)$

$\mathrm{N}-\mathrm{C}(8)-\mathrm{C}(9)-\mathrm{C}(9 \mathrm{a})$

$\mathrm{C}(7)-\mathrm{C}(8)-\mathrm{C}(9)-\mathrm{O}$

$\mathrm{C}(7)-\mathrm{C}(8)-\mathrm{C}(9)-\mathrm{C}(9 \mathrm{a})$

$\mathrm{C}(10)-\mathrm{C}(8)-\mathrm{C}(9)-\mathrm{O}$

$\mathrm{C}(10)-\mathrm{C}(8)-\mathrm{C}(9)-\mathrm{C}(9 \mathrm{a})$

$\mathrm{N}-\mathrm{C}(8)-\mathrm{C}(10)-\mathrm{C}(5)$

$\mathrm{C}(7)-\mathrm{C}(8)-\mathrm{C}(10)-\mathrm{C}(5)$

C $(9)-C(8)-C(10)-C(5)$

O-C (9)-C(9a)-C(1)

$O-C(9)-C(9 a)-C(1)$
O-C $(9)-C(9 a)-C(4 a)$

$O-C(9)-C(9 a)-C(4 a)$
$C(8)-C(9)-C(9 a)-C(1)$

$C(8)-C(9)-C(9 a)-C(1)$
$C(8)-C(9)-C(9 a)-C(4 a)$
$174.0(2) \quad 173.4(1)$

$66.7(2) \quad 173.0(1)$

$-62.2(2) \quad-64.7(2)$

$-178 \cdot 1(1) \quad-75.3(1)$

53.0(2) $\quad 47.0(2)$

$163.1(2) \quad 163.3(2)$

$42.2(2) \quad 40.8(2)$

$-78.3(2)-75.7(2)$

$-78.3(2)-75.7(2)$

$\begin{array}{rr}166.6(2) & 172.3(2) \\ -16.3(2) & -10.1(2)\end{array}$
$-143.8(2)(111.8(2)$
(1974). All calculations were performed on a Honeywell $66 / 6000$ computer or a VAX 8600 computer at the University of Kansas using programs in the KUDNA system (Takusagawa, 1984). Other experimental details are summarized in Table 1 .

Discussion. Final atomic parameters are given in Table 2 . Bond lengths, bond angles and torsion angles are listed in Table $3 .^{*}$ Views of (1) and (2) with the atomic numbering are presented in Fig. 1.

The crystal structures of biologically active norepinephrine (Andersen, 1975) and epinephrine (Carlstrom \& Bergin, 1967) have been reported with the catechol and amino group of the ethanolamine side chain in a fully extended conformation. The phenylethanolamine moiety embedded in our conformationally defined analogs (1) and (2) assumes this conformation as indicated by the torsion angles $\mathrm{N}-\mathrm{C}(8)-\mathrm{C}(9)-\mathrm{C}(9 \mathrm{a})$.

2-Amino-1-tetralols may be considered as conformationally restricted analogs of phenylethanolamine. The cyclohexene moiety in 2-amino-1-tetralol derivatives adopts a half-chair conformation with the amino group in an equatorial orientation in crystals (Motohashi, Wada, Kamiya \& Nishikawa, 1980; Hambley \& Rodgers, 1986). The 2-amino-1-tetralol moiety in (1) and (2) has a similar half-chair con-

* Lists of $\mathrm{H}$-atom coordinates, anisotropic thermal parameters and structure factors have been deposited with the British Library Document Supply Centre as Supplementary Publication No. SUP 44339 (27 pp.). Copies may be obtained through The Executive Secretary, International Union of Crystallography, 5 Abbey Square, Chester CHI 2HU, England. formation for the cyclohexene ring with the primary amine occupying an equatorial position and the hydroxyl group occupying a pseudo-equatorial one in the endo isomer (1) or a pseudo-axial one in the exo isomer (2).

Financial support from the National Institutes of Health (Grants HL34193 and GM22988) is gratefully acknowledged.

\section{References}

Andersen, A. M. (1975). Acta Chem. Scand. Ser. B, 29, 239-244. Carlstrom, D. \& Bergin, R. (1967). Acta Cryst. 23, 313-319.

Grunewald, G. L., Pleiss, M. A. \& Rafferty, M. F. (1982). Life Sci. 31, 993-1000.

Grunewald, G. L., Reitz, T. J., Hallett, A., Rutledge, C. O., Vollmer, S., ARchuletA, J. M. III \& RUTh, J. A. (1980). J. Med. Chem. 23, 614-620.

Hambley, T. W. \& Rodgers, J. R. (1986). Acta Cryst. C42, $1442-1444$.

International Tables for X-ray Crystallography (1974). Vol. IV. Birmingham: Kynoch Press. (Present distributor D. Reidel, Dordrecht.)

JOHNSON, C. K. (1976). ORTEPII. Report ORNL-5138. Oak Ridge National Laboratory, Tennessee, USA.

Main, P., Hull, S. E., Lessinger, L., Germain, G., DeclercQ, J.-P. \& Woolfson, M. M. (1978). MULTAN78. A System of Computer Programs for the Automatic Solution of Crystal Structures from X-ray Diffraction Data. Univs. of York, England, and Louvain, Belgium.

Motohashi, M., Wada, Y., KamiYa, K. \& NishikaWA, M. (1980). Chem. Pharm. Bull. 28, 3656-3661.

Rafferty, M. F. \& Grunewald, G. L. (1982). Mol. Pharmacol. 22, 127-132.

TAKUSAGawa, F. (1984). Unpublished work. Department of Chemistry, University of Kansas, Lawrence, Kansas 66045, USA. 